PREPARED FOR THE U.S. DEPARTMENT OF ENERGY, UNDER CONTRACT DE-AC02-76CH03073

PPPL-3929

PPPL-3929

UC-70

Operation of Ferroelectric Plasma Sources

in a Gas Discharge Mode

by

A. Dunaevsky and N.J. Fisch

March 2004

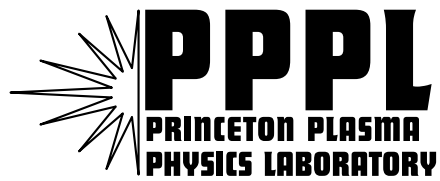

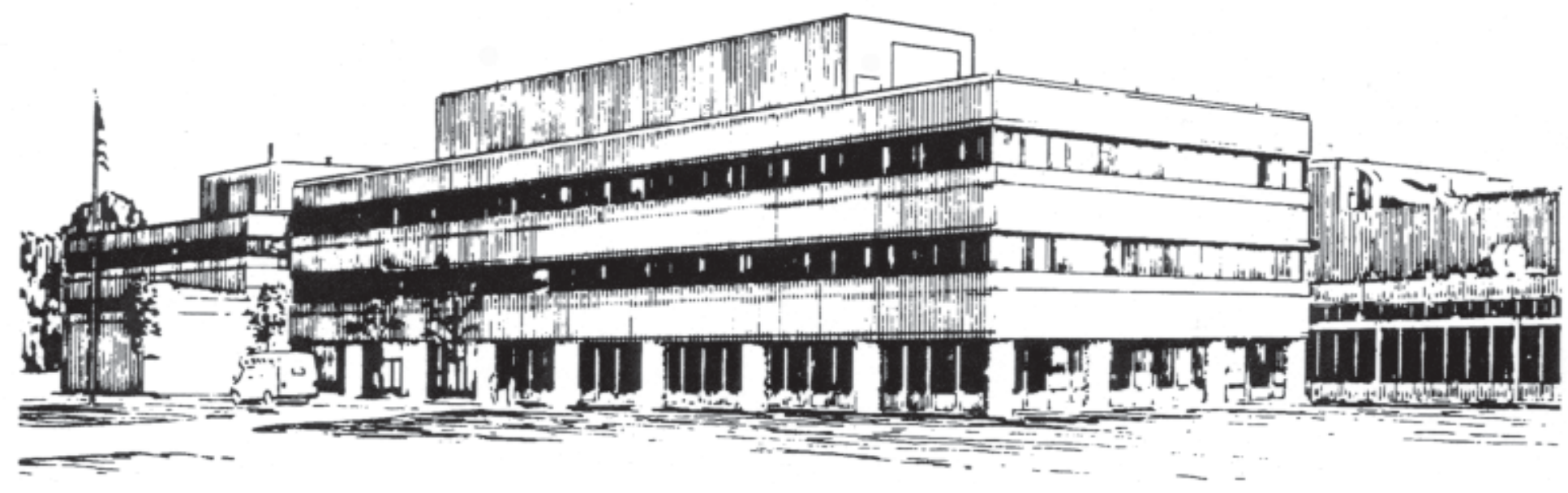

PRINCETON PLASMA PHYSICS LABORATORY PRINCETON UNIVERSITY, PRINCETON, NEW JERSEY 


\section{PPPL Reports Disclaimer}

This report was prepared as an account of work sponsored by an agency of the United States Government. Neither the United States Government nor any agency thereof, nor any of their employees, makes any warranty, express or implied, or assumes any legal liability or responsibility for the accuracy, completeness, or usefulness of any information, apparatus, product, or process disclosed, or represents that its use would not infringe privately owned rights. Reference herein to any specific commercial product, process, or service by trade name, trademark, manufacturer, or otherwise, does not necessarily constitute or imply its endorsement, recommendation, or favoring by the United States Government or any agency thereof. The views and opinions of authors expressed herein do not necessarily state or reflect those of the United States Government or any agency thereof.

\section{Availability}

This report is posted on the U.S. Department of Energy's Princeton Plasma Physics Laboratory Publications and Reports web site in Fiscal Year 2004. The home page for PPPL Reports and Publications is: http://www.pppl.gov/pub_report/

DOE and DOE Contractors can obtain copies of this report from:

U.S. Department of Energy

Office of Scientific and Technical Information

DOE Technical Information Services (DTIS)

P.O. Box 62

Oak Ridge, TN 37831

Telephone: (865) 576-8401

Fax: (865) 576-5728

Email: reports@adonis.osti.gov

This report is available to the general public from:

National Technical Information Service

U.S. Department of Commerce

5285 Port Royal Road

Springfield, VA 22161

Telephone: $1-800-553-6847$ or

(703) $605-6000$

Fax: (703) 321-8547

Internet: http://www.ntis.gov/ordering.htm 


\title{
Operation of ferroelectric plasma sources in a gas discharge mode ${ }^{a)}$
}

\author{
A. Dunaevsky ${ }^{\text {b) }}$ and N. J. Fisch \\ Princeton Plasma Physics Laboratory, Princeton University, P.O. Box 451, Princeton, New Jersey 08536
}

(Received 29 October 2003; accepted 9 February 2004)

\begin{abstract}
Ferroelectric plasma sources in vacuum are known as sources of ablative plasma, formed due to surface discharge. In this paper, observations of a gas discharge mode of operation of the ferroelectric plasma sources (FPS) are reported. The gas discharge appears at pressures between $\sim 20$ and $\sim 80$ Torr. At pressures of 1-20 Torr, there is a transition from vacuum surface discharge to the gas discharge, when both modes coexist and the surface discharges sustain the gas discharge. At pressures between 20 and 80 Torr, the surface discharges are suppressed, and FPS operates in pure gas discharge mode, with the formation of almost uniform plasma along the entire surface of the ceramics between strips. The density of the expanding plasma is estimated to be about $10^{13} \mathrm{~cm}^{-3}$ at a distance of $5.5 \mathrm{~mm}$ from the surface. The power consumption of the discharge is comparatively low, making it useful for various applications. This paper also presents direct measurements of the yield of secondary electron emission from ferroelectric ceramics, which, at low energies of primary electrons, is high and dependent on the polarization of the ferroelectric material.

(C) 2004 American Institute of Physics. [DOI: 10.1063/1.1692162]
\end{abstract}

\section{INTRODUCTION}

Ferroelectric plasma sources (FPS) are known as vacuum sources of the surface discharge plasma. ${ }^{1}$ Imagine a bulk of ferroelectric ceramics placed in vacuum and covered by two electrodes, rear solid and front patterned, like a capacitor. Numerous surface discharges will appear along the edges of the front electrode pattern in response to the application of a driving pulse with sufficient amplitude. ${ }^{2}$ The density of the surface discharge plasma, measured at about $1 \mathrm{~cm}$ from the surface, reaches $10^{11}-10^{12} \mathrm{~cm}^{-3}$, and the electron temperature is $2-3 \mathrm{eV}$. The plasma consists of ions from the compounds of ceramic material, and expands from the surface with the velocity of $1-1.5 \mathrm{~cm} / \mu \mathrm{s}$. The attractiveness of vacuum FPS is in the virtually unlimited area of the source, which can have an arbitrary shape, and in the compatibility with vacuum systems operating at $10^{-6}-10^{-5}$ Torr. These features supported wide application of FPS as plasma cathodes for various microwave devices. ${ }^{3}$

The principle of FPS operation is based on the high polarizability of ferroelectrics. ${ }^{4}$ Application of the driving pulse leads to appearance of substantial polarization charge at the front surface. Pattern geometry of the front electrode causes nonuniformity in distribution of the polarization charge along the surface, which means the existence of a tangential electric field. Enhanced at triple junctions at the edges of the front electrode pattern, the electric field initiates field emission. Field emission electrons ignite secondary electron avalanches along the ceramic surface in the tangential electric field. Avalanching electrons bombard the surface, which leads to intense neutral desorption. Ionization of the desorbed neutrals results in the formation of a plasma channel.

As described, the role of ferroelectrics appears to be lim-

\footnotetext{
a) Paper RI 2 5, Bull. Am. Phys. Soc. 48, 294 (2003).

${ }^{\mathrm{b})}$ Invited speaker.
}

ited by effects related to electric fields. Indeed, these effects seem dominant in the physics of FPS. However, other properties of ferroelectrics could play an important role as well. For instance, the secondary electron emission properties of ferroelectrics may affect electron avalanches, and therefore the plasma density. "Weak" ferroelectric emission ${ }^{5}$ may ignite avalanches, even if they are not adjacent to the edges of the front electrode. The possible role played by these effects, however, has not yet been recognized.

In the plasma of surface discharges, probe measurements of plasma parameters are possible only at a distance, in the expanding plasma. ${ }^{1}$ Recent spectroscopic studies ${ }^{6,7}$ did not allow retrieving unambiguous values of both initial values of the plasma density and the electron temperature in the channel, or stem, of the discharge. Recently, a method of deducing of the initial plasma parameters from temporal profiles of the density of expanding plasma has been developed. ${ }^{8}$ It was shown that the initial plasma density in the stem of the discharge could reach $(4-40) \times 10^{16} \mathrm{~cm}^{-3}$, and the initial electron temperature is $16-20 \mathrm{eV}$. Such a high density corresponds to a pressure of neutral gas of 1-10 Torr at room temperature, assuming full ionization. It indeed corresponds within an order of magnitude to the estimation of neutral density at the surface reported by Chirko et al. ${ }^{9}$ Assuming the thickness of the discharge stems as 10-20 $\mu \mathrm{m}$, we obtain an initial density of neutrals $\geqslant 10^{17} \mathrm{~cm}^{-3}$. $^{8}$

Thus, it is reasonable to expect the appearance of a king of gas discharge at the surface of FPS immersed into a gas with a density of several Torr or higher. Indeed, a gas discharge induced by FPS was observed earlier. Teranishi $e t$ al. reported about a luminous phenomenon on a piezoelectric transformer in different gases at a pressure of $0.08-5$ Torr. ${ }^{10,11}$ Light emission from a corona-like discharge was observed at pressures of 0.008-2.2 Torr in pseudospark switches triggered by ferroelectric cathodes. ${ }^{12,13}$ It confirms 
the expectation to find a gas discharge mode of FPS operation at pressures in the range of a few Torr.

In this paper, we report observations of the gas discharge mode of FPS operation. This mode appeared at background pressures of 1-80 Torr; at lower and higher pressures the surface discharge mode dominates. We estimate the plasma density and electron temperature in the gas discharge. Evidence of the role of "weak" ferroelectric emission was observed in the gas discharge mode. In addition, we present the results of our measurements of secondary electron emission for ferroelectrics, induced by low energy electrons. The yield of secondary electron emission in the low energy range is high and depends on material polarization.

\section{SECONDARY ELECTRON EMISSION FROM FERROELECTRICS}

The yield of electron-induced secondary electron emission (SEE) is an important parameter in plasma-wall interaction. It determines the balance of fluxes, and consequently the potential drop on the plasma sheath. ${ }^{14}$ For low temperature plasmas, primary electrons reaching the wall will have energies lower than $100 \mathrm{eV}$. Thus, for sheath effects, the scope of interest is the range of primary energies $\leqslant 100 \mathrm{eV}$.

Parameters of the surface discharge are determined by the secondary emission properties of dielectric material. Indeed, the initial stage of the surface flashover is the development of a saturated SEE avalanche. ${ }^{15}$ The SEE avalanche is assumed saturated if the current of avalanching electrons to the surface is equal to the outgoing current of secondary electrons, or when the total yield of SEE $\delta=1$ is realized. Thus, the avalanching electrons strike the surface with energy $\xi_{1}$ which corresponds to the unit yield of SEE. For a plane dielectric surface, with applied tangential electric field $E_{\|}$, electrons, which are released for the surface with the mean initial energy of $\xi_{0}$, reach a height: ${ }^{16}$

$$
x_{m} \approx \frac{\xi_{0}}{E_{\perp}}=\frac{\sqrt{\xi_{0}\left(\xi_{1}-\xi_{0}\right)}}{\sqrt{2} E_{\|}}
$$

and travel along the surface:

$$
y_{m} \approx \frac{4 \xi_{0} E_{\|}}{E_{\perp}^{2}}=\frac{2\left(\xi_{1}-\xi_{0}\right)}{E_{\|}} .
$$

Here $E_{\perp}$ is the normal component of the electric field, which appears between the negative space charge of the electron avalanche and the positive charge of the dielectric surface. All values are in SI, except energies $\xi_{1}$ and $\xi_{0}$, which are in $\mathrm{eV}$. The surface acquires a positive surface charge:

$$
\sigma_{+}=2 \varepsilon_{0} E_{\perp},
$$

which is assumed equal to the negative charge $\sigma_{-}$of the electron avalanche. ${ }^{16}$ It was shown ${ }^{17}$ that, assuming a cosine distribution of released electrons, the equilibrium in surface charge is reached when the angle $\theta$ between the electric field and the surface is equal to

$$
\operatorname{tg}(\theta)=\frac{E_{\perp}}{E_{\|}}=\sqrt{\frac{2 \xi_{0}}{\xi_{1}-\xi_{0}}} .
$$

The current density of the avalanching electrons returning to the surface can be found as: ${ }^{17}$

$$
j_{\perp} \sim \frac{\sigma_{-}\left\langle v_{e}\right\rangle}{y_{m}} .
$$

Here $v_{e} \sim\left(e \xi_{1} / 2 m_{e}\right)^{1 / 2}$ is the average drift velocity of electrons in the avalanche. The rate of desorption of neutrals, $\Gamma_{n}$, from the surface bombarded by electrons with the current density $j_{\perp}$, depends on the desorption efficiency $\gamma,{ }^{17}$

$$
\Gamma_{n}=\gamma \frac{j_{\perp}}{e} .
$$

Thus, the properties of the dielectric material like the desorption efficiency $\gamma$, the initial energy $\xi_{0}$ of the secondary electrons, and the energy $\xi_{1}$ of the unity yield of SEE, will determine the desorption rate $\Gamma_{n}$,

$$
\Gamma_{n}=\frac{\gamma \varepsilon_{0} E_{\|}^{2}}{\left(\xi_{1}-\xi_{0}\right) \sqrt{e m_{e}}} \sqrt{\frac{\xi_{1} \xi_{0}}{\xi_{1}-\xi_{0}}} .
$$

While the initial energy $\xi_{0}$ of the secondary electrons is usually a few $\mathrm{eV}$ and does not change significantly from one material to another, variations of $\gamma$ and $\xi_{1}$ could considerably affect $\Gamma_{n}$. The higher the desorption rate, the faster is the growth of neutral density in the volume of the electron avalanche. High yield of SEE in the low energy region, which corresponds to low $\xi_{1}$, can result in higher $\Gamma_{n}$ and consequently in faster transition to plasma formation and higher plasma density in the discharge.

In spite of its importance, the actual yield of SEE in the low energy region has not been studied for most modern dielectric materials. Indeed, measurements of SEE from dielectrics are complicated because of surface charging effects. The surface will acquire a positive charge if the flux of secondary electrons is higher than the primary flux, and negative in the opposite case. The surface charge affects strongly the actual yield of secondary electrons. Thus, special steps have to be taken to minimize the surface charging and to remove the accumulated charge.

Recently, we developed a setup to measure the total yield of electron induced SEE from dielectrics at energies of primary electrons from 6 to $1000 \mathrm{eV}{ }^{18}$ The primary electron beam had a current of $\sim 500 \mathrm{nA}$ with the duration of $\sim 2 \mu \mathrm{s}$. The diameter of the beam focal spot was $1 \mathrm{~mm}$. The samples can be heated up to $700{ }^{\circ} \mathrm{C}$. The procedure of the surface charge removal after each shot of primary electron beam was based on cleaning by a volatile conducting solvent followed by heating at $150-200^{\circ} \mathrm{C}$ in vacuum of about $10^{-7}$ Torr. This procedure did not provide complete removal of the surface charge, which is accumulated inside the material on the depth of several atomic layers, and the deviation of the SEE yield was about $10 \%$.

Since then, the performance of the setup has been improved by several modifications. The direct coupled amplifier was substituted by another one with 10 times higher amplification, which allowed the primary electron current to decrease to $100 \mathrm{nA}$ and the duration to decrease to $1 \mu \mathrm{s}$. The focal spot diameter was increased from $1 \mathrm{~mm}$ to $2.5 \mathrm{~mm}$ by the use of new beam collimator. This increase corresponds to 


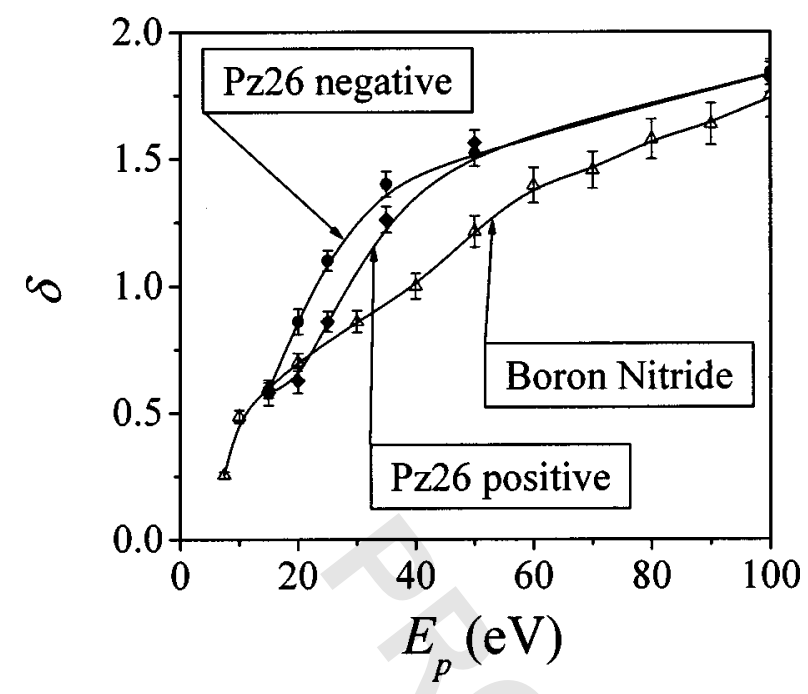

FIG. 1. Total yield of electron induced secondary electron emission from Pz26 ferroelectric ceramics for negative and positive polarizations, and from boron nitride.

more than 6 times lower density of the induced surface charge. Faraday cup for measurements of primary current and rotary positioning system were modified as well. The procedure of the surface charge removal was changed by using a beam of neutral plasma. The tolerance in the energy of primary electrons was less than $0.1 \mathrm{eV}$. The overall precision of the measurements was improved to $<5 \%$.

On this improved setup, we performed preliminary measurements of the yield of SEE from ferroelectrics. We studied a commercially available material Pz26 produced by Ferroperm. The yield of SEE was measured from poled samples for both negative and positive polarizations. The results are shown in Fig. 1, together with the corresponding data for boron nitride for comparison.

At energies of primary electrons between 20 and $100 \mathrm{eV}$, the yield of SEE from Pz26 was found considerably higher than the one from boron nitride and other conventional dielectric materials like alumina ceramics, Macor, and Teflon. The unity yield of SEE from Pz26 corresponds to the energy of primary electrons of $\xi_{1} \sim 20-30 \mathrm{eV}$, while for boron nitride $\xi_{1}=40 \mathrm{eV}$. Moreover, between 15 and $45 \mathrm{eV}$ we observed a difference in the SEE yield between two polarizations of the Pz26. The unity yield of SEE was found at $\xi_{1}$ $=22 \mathrm{eV}$ for the negative polarization of Pz26 samples and at $\xi_{1}=28 \mathrm{eV}$ for the positive polarization. Assuming the desorption efficiency and the initial energy of secondary electrons does not depend on polarization, the observed difference results in 1.3 higher desorption rate for negatively poled Pz26, as it follows from Eq. (7). This difference in the desorption rate for positively and negatively poled material might explain the difference in the plasma density for different polarizations. ${ }^{1,2}$

\section{GAS DISCHARGE MODE OF FPS OPERATION}

The condition of appearance of the gas discharge mode of FPS operation and measurements of the plasma parameters of the gas discharge were performed on the experimen-

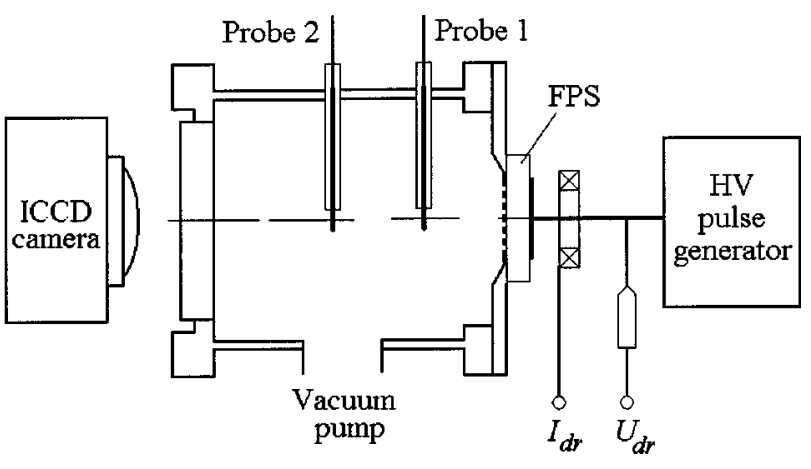

FIG. 2. Experimental setup for study of the gas discharge mode.

tal setup shown in Fig. 2. The ferroelectric plasma source employed a disk of ferroelectric ceramics APC-850 produced by American Piezo Ceramics. The disk had a diameter of 38 $\mathrm{mm}$ and a thickness of $2 \mathrm{~mm}$. The grounded front electrode had a pattern in form of strips interconnected by an outer copper ring with a diameter of $20 \mathrm{~mm}$, which was glued to a brass flange by a conducting epoxy. The brass flange was connected to a chamber filled by a gas. The majority of experiments were done with argon, however, a gas discharge mode was observed with air and xenon as well. The pressure in the chamber was measured by MKS Baratron capacitive manometer.

A negative driving pulse with a duration of $\sim 500 \mathrm{~ns}$ FWHM was supplied to a solid rear electrode by a Blumlein pulse generator with a repetition rate of $10-100 \mathrm{~Hz}$. The amplitude of the driving electric field was varied from 3 to $30 \mathrm{kV} / \mathrm{cm}$. The driving voltage was measured by LeCroy high voltage divider; the current supplied to the FPS was measured by Pearson Rogowskii Coil.

The parameters of the expanding plasma were estimated from measurements by a pair of biased probes, similarly to Ref. 1. The probes were placed at $5.5 \mathrm{~mm}$ and at $14 \mathrm{~mm}$ from the front surface of the FPS (see Fig. 2). The probes were mounted with an azimuthal displacement of $90^{\circ}$ to prevent screening of the second probe by the first one. The probes were made of tungsten wire with a diameter of 1.5 $\mathrm{mm}$ and were immersed in the chamber through Wilson seals.

Fast frame imaging of FPS operation in the gas discharge mode was carried out by Andor 1-Star® ICCD camera. The images were taken with the frame duration of 400 ns, with the frame beginning simultaneously with the front of the driving pulse. A set of fast frame images of the discharge in argon at the driving pulse amplitude of $4 \mathrm{kV}$ is shown in Fig. 3. From the vacuum of $10^{-7}$ Torr to about $0.5-1$ Torr, the surface discharges looked almost unchanged, as illustrated by Fig. 3(a).

At pressures higher than 1 Torr, the luminosity of the discharge grows rapidly and reaches maximum at 7-10 Torr. The typical stems of the surface discharges still exist, but became almost irresolvable on the bright background light appeared above the ceramic surface between the strips of the front electrode. The typical image of the discharge at $P$ $=8$ Torr is shown in Fig. 3(b). We associate this light emission with plasma formed due to ionization of the background 


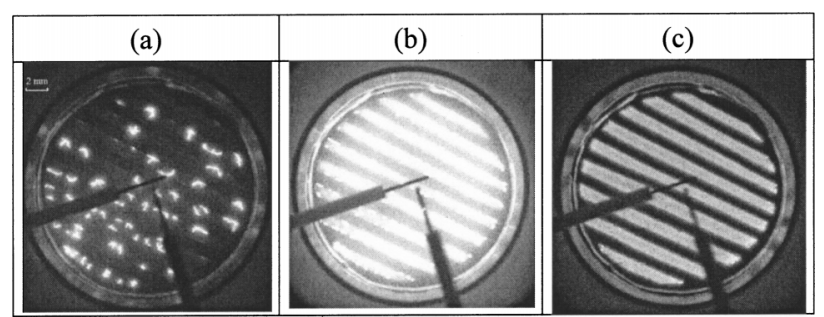

FIG. 3. Fast frame imaging of the light emission from FPS at (a) 0.2 Torr (b) 8 Torr, and (c) 30 Torr of argon.

gas. Similar images were observed also by Chirko et al., ${ }^{9}$ where a FPS in vacuum was stressed by a pair of driving pulses with a proper delay between them. By our present results, we could confirm the supposition made in Ref. 9 regarding the nature of the plasma density enhancement. Indeed, the discharge induced by the second driving pulse may happen in the layer of gas desorbed during the first firing cycle.

The surface discharges in the pressure range of 1-20 Torr were observed by filtering out the light emission from the gas discharge. In general, the complete removal of the gas discharge spectra can be done only by filtering out the entire spectra of the argon neutrals and ions from the image. However, the wavelength of the majority of ArI and ArII intense lines are shorter than $400 \mathrm{~nm}$, while ceramic materials $(\mathrm{Pb}, \mathrm{Zr}$, Ti) have many intense lines in longer wave length range. Thus, it appears possible to suppress sufficiently the background from the gas discharges by filtering out the short-wavelength light. It was done by a combination of a gray filter with an UV filter. Such a combined filter allows the bright points to be visualized clearly.

The typical filtered image is presented in Fig. 4 for $P$ $\sim 8.5$ Torr. The shape of the surface discharge stems is changed to localized bright points, which are not always adjacent to the edges of the front electrode. Moreover, on virtually every image, a few points were found not to be adjacent to the edges of the front electrode, but in the middle

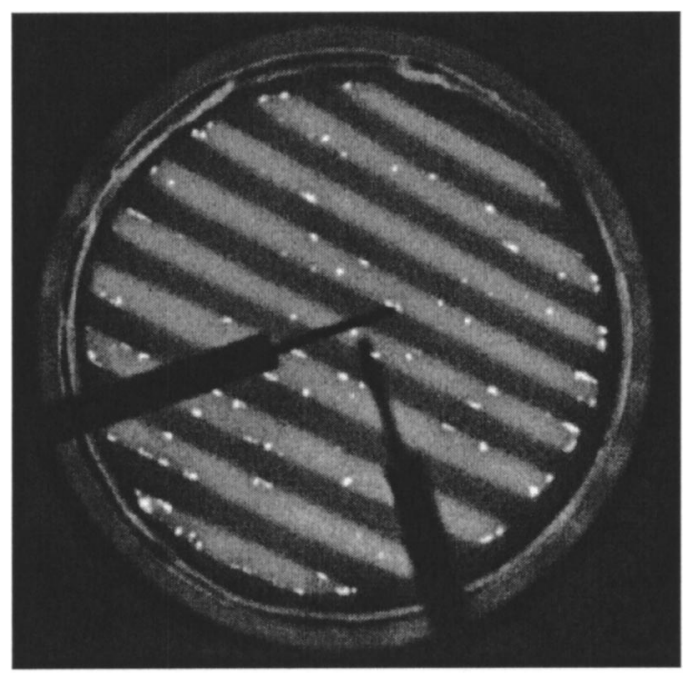

FIG. 4. Fast frame imaging of the light emission from FPS at 8 Torr with filtered out light from Ar discharge. between the strips. These avalanche stems cannot be ignited by field emission from the triple points, as it suggested in the scenario of FPS operation. The appearance of these discharge stems is not delayed with respect to the others, so we could not suppose these discharges were induced by "primary" discharges at triple points.

The observed effect might be explained by influence of the "weak" ferroelectric emission..$^{5}$ Indeed, this low-current emission could happen from the entire surface of the ceramics between the strips, and could ignite the discharge at arbitrary points. In vacuum, all discharges have to be closed to the front electrode, and the seeds of avalanches were irresolvable. In the presence of gas discharge plasma, seeds which are not adjacent to the edges of the front electrode may initiate discharges similar to the unipolar arc. The appearance of such discharges conditioned the possibility to make a supposition regarding the role of the "weak" ferroelectric emission in the FPS operation.

At higher pressures, the luminosity of the discharge decreases, and at pressures above 20 Torr, evidence of the formation of surface discharges disappear completely. The discharge seems almost uniform along the entire surface of the ceramics between strips, in contrast with uncovered middle region between the strips in the surface discharge mode [see Fig. 3(c)]. Later growth of pressure leads to the gradual decrease of the discharge luminosity, to the appearance of the dark region in the middle between the strips, and finally to the reappearance of the surface discharges, as it was observed in air at atmospheric pressure. ${ }^{2}$

Visual light imaging of the discharge indicates the existence of two different regimes of the gas discharge mode. At the pressure range between a few Torr and about 20 Torr, the discharge appears in a combined form of surface and gas discharges. At higher pressures, pure gas discharge is realized.

Plasma parameters have been estimated from the measurements by two biased probes, placed at $5.5 \mathrm{~mm}$ and 11 $\mathrm{mm}$ from the FPS surface. In contrast with the surface discharge mode in vacuum, expansion of the plasma layer outward the surface is not collisionless but rather diffusive. In this case, the problem of deducing the initial plasma parameters in the layer near the surface requires a solution of the collisional Vlasov equation. Without a simple analytic solution, the method of the deducing of initial plasma parameters described in Ref. 8 cannot be directly used, and only distant measured values can be obtained.

A typical signal from the first probe, observed at $P$ $=8$ Torr of Ar, is presented in Fig. 5 together with the traces of the driving voltage, $U_{\mathrm{dr}}$, and the driving current, $I_{\mathrm{dr}}$. Temporal behavior of the probe current coincides with the luminosity of the discharge observed by the fast frame imaging. At pressures higher than 1 Torr, the collisionless probe theory is inapplicable. Indeed, at the background pressure of about 5 Torr, the mean free path of electron-neutral collisions is $\lambda_{n} \sim 2 \times 10^{-3} \mathrm{~cm}$, that is much smaller than the probe radius $R_{p}=250 \times 10^{-3} \mathrm{~cm}$. Estimation of the sheath thickness $^{19}$ yields $h \sim 0.3 \times 10^{-3} \mathrm{~cm}$. Thus, we should suppose that at $P \sim 5$ Torr $R_{p} \gg \lambda_{n} \gg h$. This means that the sheath is thin and collisionless, and the disturbance of the 


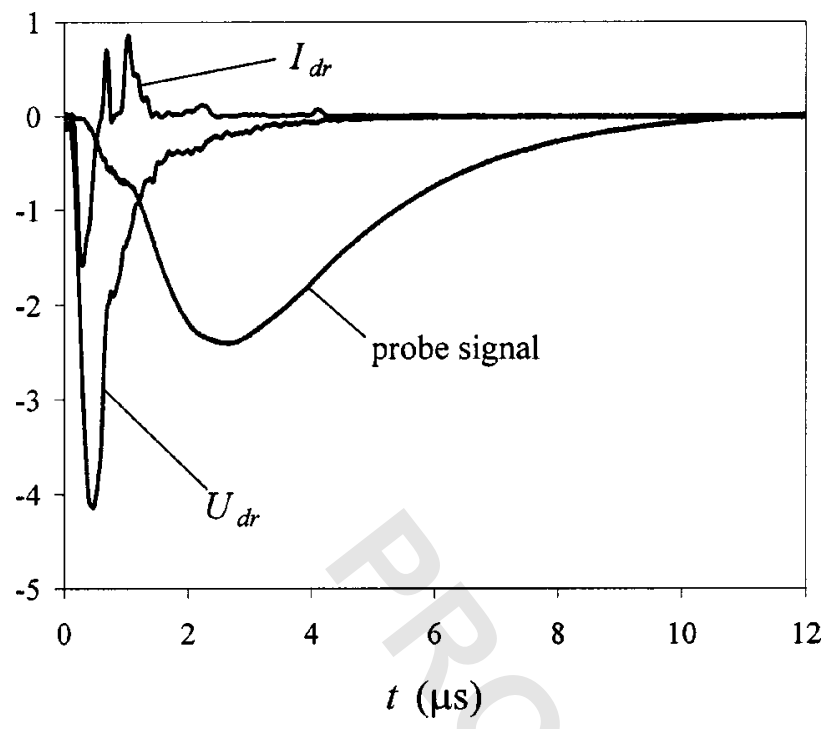

FIG. 5. Temporal behavior of the driving voltage, the driving current, and the signal from biased probe placed at $5.5 \mathrm{~mm}$ from the FPS surface.

drift motion of electrons and ions outside the sheath should be insufficient.

In spite of collisional regime, the analysis of the slope of electron branch of the probe $A-V$ characteristics still can yield an estimation of the electron temperature. ${ }^{20}$ Such estimation for temporal behavior of the electron temperature at two probes locations is shown in Fig. 6. The temperature decreases fast in time, and it seems the initial value might be higher that $10 \mathrm{eV}$. Some decrease is observed also with the distance from the surface.

The high initial electron temperature might be explained by the nature of the transient process of combined surface and gas discharge. Indeed, at the initial stage of the surface discharge, electrons in the saturated secondary emission avalanche have a mean energy of $\sim\left(\xi_{0}+\xi_{1}\right) / 2$. According to our measurements (see Sec. II), for Pz26 $\xi_{1} \sim 20-30 \mathrm{eV}$ depending on the polarization of the material. Assuming $\xi_{1}$ $\sim 2-3 \mathrm{eV}$, the mean electron energy is about $11-16 \mathrm{eV}$. This

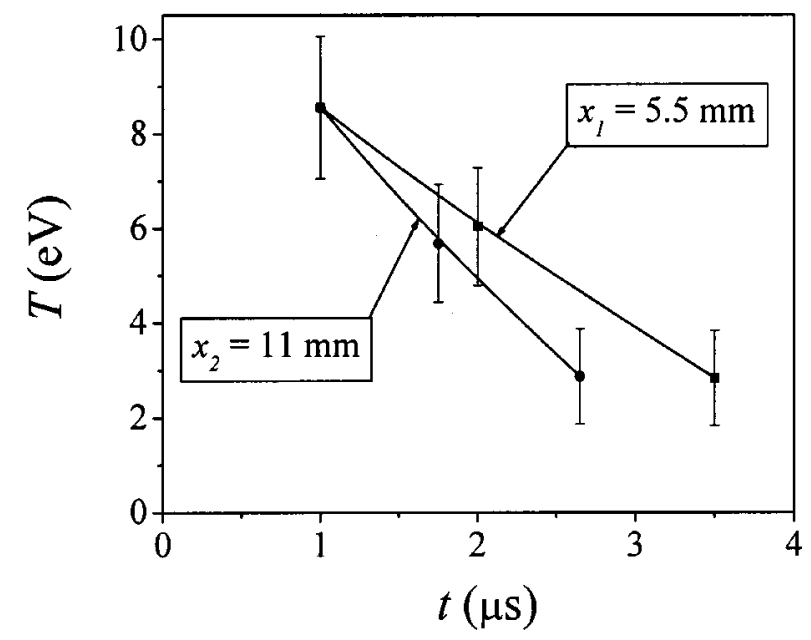

FIG. 6. Temporal behavior of the electron temperature measured in expanding plasma at two distances from the FPS surface. mean energy may correspond to the initial electron temperature in the surface discharge. Notice the same order of initial electron temperature in the stem of the surface discharge on ferroelectric plasma cathodes in vacuum, which was measured in Ref. 8. The concept of temperature is applicable here, because at the gas pressure of about 10 Torr the frequency of momentum transfer collisions is high, $\nu_{m} \sim 4$ $\times 10^{10} \mathrm{~s}^{-1}$, and randomization of the energy gained by electrons from the driving electric field happens fast.

The portion of energy transferred by electrons in momentum transfer collisions in $\mathrm{Ar}$ at $P \sim 10$ Torr is $\sim 3$ $\times 10^{-5} T_{e}$, which corresponds $\sim 0.4 \mathrm{eV}$ over the duration of the driving pulse $\sim 0.4 \mu \mathrm{s}$. However, taking into account high frequency of the ion/neutral momentum transfer collisions (both elastic and charge exchange) $\nu_{M} \sim 3 \times 10^{8} \mathrm{~s}^{-1}$, the temperature of ions at the probe location can be assumed about of temperature of the background gas $T_{i} \sim T_{n}$ $\sim 300 \mathrm{~K}$.

The plasma density can be estimated roughly from the ion saturation current measured by the probes. At high densities of the background gas, the ion saturation current can be derived from a solution of the diffusive motion of ions in the probe presheath. For a very rough estimation, however, a correction on the ion mobility can be made in the ion saturation current

$$
i_{\text {ion }}^{\text {sat }} \sim I_{\text {ion }}^{\text {sat }} \sqrt{\frac{T_{e}}{T_{i} R_{p}}} .
$$

Here $\lambda_{\text {ion }}$ is the mean free path of ions, and $I_{\text {ion }}^{\text {sat }}$ is the ion saturation current in the collisionless case. This estimation of the plasma density based on the measured ion current yields $n_{i}<(4-8) \times 10^{13} \mathrm{~cm}^{-3}$ at $x=5.5 \mathrm{~mm}$ from the surface, $t$ $=2.2 \mathrm{~ms}$ from the beginning of the driving pulse, $U_{\mathrm{dr}}$ $=4 \mathrm{kV}$, and $P=5$ Torr of Ar. At $x=11 \mathrm{~mm}$, the density is much lower, $n_{i} \leqslant 10^{11} \mathrm{~cm}^{-3}$, and could not be determined confidently from the ion saturation current because of very low ion current to the probe. The observed gradient of the plasma density indicates in linear approximation the possible initial plasma density near the surface in order of $10^{15} \mathrm{~cm}^{-3}$.

The measured dependence of the probe current on the background pressure $P$ is presented in Fig. 7 for air and argon, at $U_{\mathrm{dr}}=4 \mathrm{kV}$. The maximum of the probe current for argon corresponds roughly to $n_{i} \sim 6 \times 10^{13} \mathrm{~cm}^{-3}$ and is shifted toward the higher pressures comparing to air. One can see that the probe signal for argon is 1.5-2 times larger than for air in the pressure range of 3-20 Torr and more than 5 times larger at higher pressures. The observed pressure dependence for Ar and air is similar to the Paschen law for the breakdown voltage $V_{b}$ of a gap $d$ filled by a gas with a pressure $P:^{20}$

$$
V=\frac{B P d}{\ln (A P d)-\ln (1+1 / \gamma)} .
$$

Here $A=12$ (cm Torr) $^{-1}$ and $B=180 \mathrm{~V} /(\mathrm{cm}$ Torr) for argon; $A=15$ (cm Torr) $^{-1}$ and $B=365 \mathrm{~V} /$ (cm Torr) for air; ${ }^{20} \gamma$ was assumed $\sim 0.1$; $d$ roughly corresponds to the half width of the gap between strips of the front electrode. Thus, the mechanism of the discharge should be similar to the Paschen 


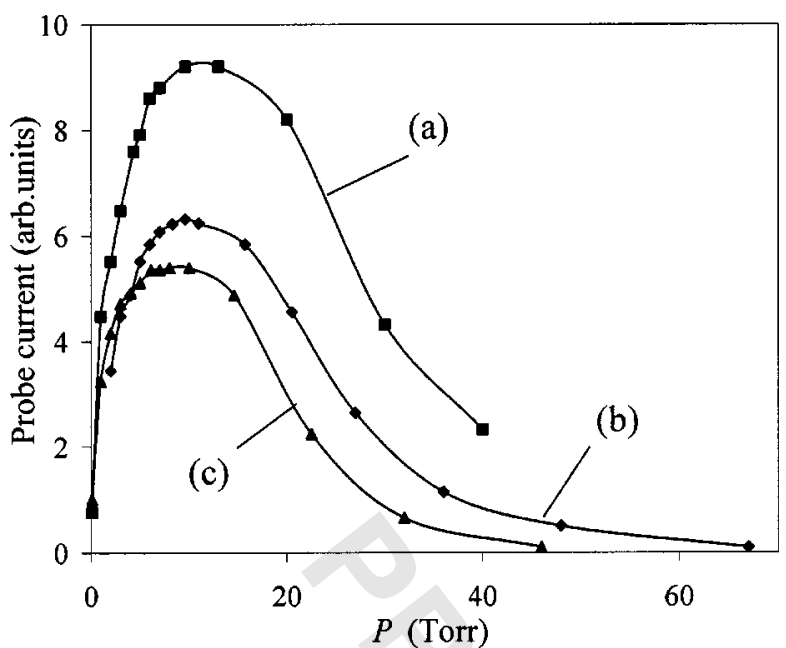

FIG. 7. Dependence of the amplitude of the probe signal on the gas pressure, for (a) argon, (b) air at $1 \mathrm{~mm}$ strips width, and (c) air at $2 \mathrm{~mm}$ strips width. The probe is placed at $5.5 \mathrm{~mm}$ from the FPS surface. Plasma density in argon at $P \sim 8$ Torr is estimated as $n_{i} \sim(4-8) \times 10^{13} \mathrm{~cm}^{-3}$.

breakdown. Indeed, changes in pattern geometry of the front electrode lead to correspondent changes in the pressure dependence. In air, for instance, twice wider strips and twice wider gaps between strips $(2 \mathrm{~mm}$ instead of $1 \mathrm{~mm}$ as in all other experiments) causes about 7\% higher plasma density and slight shift toward higher pressures (see Fig. 7), in agreement with Eq. (9) for the wider gap $d$.

The observed dependence should be also considered as an experimental evidence of the pressure range of the desorbed gases in the stem of the surface discharge on ferroelectrics. Indeed, Avdienko and Malev ${ }^{21}$ from their model of the surface discharge estimated the equivalent number density of neutrals about $n_{0} \sim 1.5-4 \times 10^{18} \mathrm{~cm}^{-3}$ (equivalent pressure of about 50-100 Torr). Bugaev et al. ${ }^{22}$ suggested slightly lower density $n_{0} \sim 0.2-1 \times 10^{18} \mathrm{~cm}^{-3}$ (equivalent pressure of about 5-30 Torr). Gray, ${ }^{23}$ however, cites several estimations in the range of $n_{0} \sim 10^{19} \mathrm{~cm}^{-3}$. Visual light imaging shows that the formation of stems of the surface discharges disappear at $P \sim 20$ Torr. This pressure could correspond to reaching the optimal conditions for ionization, when additional desorption from the surface will not cause significant growth of the plasma density. The estimations made by Bugaev et al. seem close to our present results, and the neutral density in the stem of the surface discharge is about $n_{0}$ $\sim 0.2-1 \times 10^{18} \mathrm{~cm}^{-3}$.

Analysis of the energy consumption of the discharge also reveals indirect evidence of the optimal conditions at $P$ $\sim 20$ Torr. Pressure dependence of the total energy supplied to the discharge, found as a time integrated product of the driving voltage and the driving current, is shown in Fig. 8 for the driving voltage amplitude of $4 \mathrm{kV}$. From vacuum of about $10^{-7}$ Torr to $P \sim 1.5$ Torr, when the FPS operated in the surface discharge mode, the energy supplied to the discharge in a single pulse is about $\sim 1.9 \mathrm{~mJ}$ and is almost unchanged. At higher pressures, when FPS operates in the combined gas and surface discharge mode, the supplied energy grows gradually up to $\sim 2.5 \mathrm{~mJ}$ at $P \approx 20$ Torr. In the gas discharge mode, at $P \geqslant 20$ Torr, the supplied energy re-

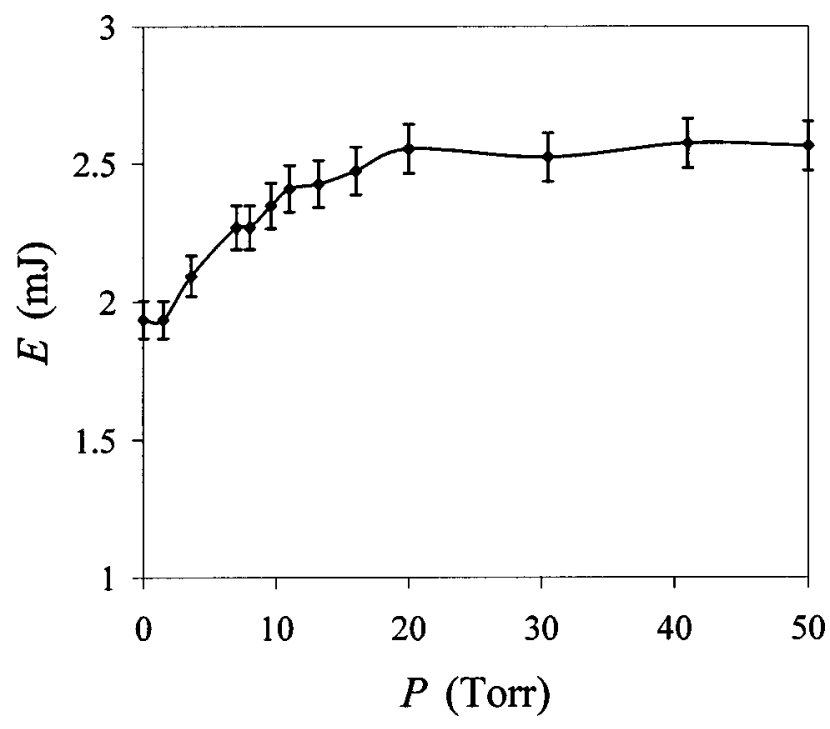

FIG. 8. Energy supplied to the discharge in a single pulse; $U_{\mathrm{dr}}=4 \mathrm{kV}$.

mains rather constant up to $P \sim 80$ Torr and decreases at higher pressures. Thus, at $P$ between 20 and 80 Torr the power dissipation in the discharge is maximal, which may correspond to the optimal discharge conditions.

The efficiency of the FPS operation in combined surface and gas discharge mode is considerably higher than in vacuum surface discharge mode. Indeed, at the position of the first probe, $x=5.5 \mathrm{~mm}$ from the surface, at $t=2.2 \mathrm{~ms}$ from the beginning of the driving pulse, plasma density in the combined mode at $P=8$ Torr was found about of order of magnitude higher than in vacuum discharge mode. ${ }^{8}$ Difference in the energy consumption between $P \sim 10^{-7}$ Torr and $P=8$ Torr at the same driving voltage amplitude is only $\sim 20 \%$.

The neutral density $n_{0} \sim 0.2-1 \times 10^{18} \mathrm{~cm}^{-3}$ agrees with the measurements of the initial plasma density in the stem of the surface discharge, reported in Ref. 8. The initial plasma density was found to be $n_{i} \sim 0.4-4 \times 10^{17} \mathrm{~cm}^{-3}$, which would correspond to an ionization rate of $\sim 20 \%$. Masten et al. ${ }^{24}$ performed estimating measurements of the plasma density by deflection of a laser beam, and also found the initial density in the stem of the discharge in the range of $10^{17} \mathrm{~cm}^{-3}$.

The ion and electron currents on the probes were found to depend on the amplitude of the driving voltage, as it shown in Fig. 9 for $P=5$ Torr. For both Ar and air, the dependence is close to linear with saturation at higher driving amplitudes. The same behavior of the plasma density was observed for the surface discharge mode. This could mean that at pressures of 1-20 Torr, when both surface and gas discharges are observed, the surface discharge sustains the gas discharge, and the total plasma density is determined by the plasma density in the surface discharges.

\section{SUMMARY}

Ferroelectric plasma sources can operate in two modes: surface discharge and gas discharge. Gas discharge mode appears at pressures of $20-80$ Torr. At pressures of 1-20 


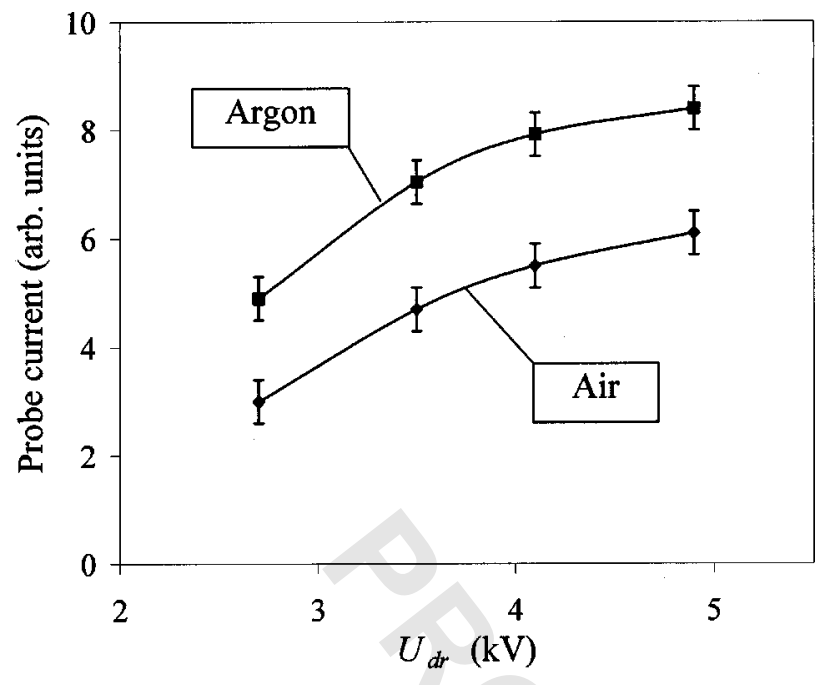

FIG. 9. Amplitude of the probe signal vs the amplitude of the driving pulse for argon and air, at $P=5$ Torr.

Torr, there is a transition from vacuum surface discharge to the gas discharge, and the surface discharge in this pressure range sustains the gas discharge. At pressures between 20 and 80 Torr, surface discharges are suppressed, and FPS operates in pure gas discharge mode, with the formation of almost uniform plasma along the entire surface of the ceramics between strips. The threshold of the gas discharge mode is several times less than the threshold of the surface discharge mode.

Maximal density of the expanding plasma in the combined surface and gas discharge mode is estimated as $(4-8) \times 10^{13} \mathrm{~cm}^{-3}$ at the distance of $5.5 \mathrm{~mm}$ from the surface. Electron temperature at $1 \mu$ s from the beginning of the driving pulse is about of $8-9 \mathrm{eV}$ and decreases with time and distance from the FPS surface. Initial electron temperature at the surface of FPS could be estimated about or higher than $10 \mathrm{eV}$ and the density at least $10^{15} \mathrm{~cm}^{-3}$.

The pressure range of 5-30 Torr, at which the transition from surface to gas discharge mode is realized, should correspond to the pressure in the channel of the surface discharge in vacuum. The neutral density of about $10^{18} \mathrm{~cm}^{-3}$ agrees well with estimations of other authors and corresponds to reasonable ionization rate of $\sim 20 \%$. The pressure dependence of the plasma density indicates that Paschen breakdown should be a possible mechanism of the gas discharge mode of FPS.

A preliminary study of secondary electron emission from ferroelectrics shows that at low energies of primary electrons the total yield of electron-induced SEE from ferroelectrics is high comparing with other dielectrics and it depends on polarization of the material. Low value of energy which corresponds to the unity yield of SEE from ferroelectrics leads to higher desorption rate and consequently to lower threshold of the surface discharge on ferroelectrics. In the combined gas and surface discharge mode, electron avalanches not adjacent to the edges of the front electrode were observed. These avalanches might be ignited not by field emission from triple junctions but also by "weak" ferroelectric emission.

The efficiency of FPS in the gas discharge mode is higher than in the vacuum surface discharge. Taking into account possibly longer lifetime, gaseous FPS can be promising for creation of layers of uniform and dense plasma on surfaces with large area and arbitrary shape, which is important for some aerospace applications.

\section{ACKNOWLEDGMENTS}

We gratefully acknowledge Dr. Y. Raitses for very fruitful discussions. We also thank Richard Yager and Gary d'Amico for technical support.

This work was supported by the New Jersey Commission of Science and Technology, and by the DOE under Contract No. DE-AC02-76-CH03073.

${ }^{1}$ A. Dunaevsky, Ya. E. Krasik, J. Felsteiner, and S. Dorfman, J. Appl. Phys. 85, 8464 (1999).

${ }^{2}$ Ya. E. Krasik, A. Dunaevsky, and J. Felsteiner, J. Appl. Phys. 85, 7946 (1999).

${ }^{3}$ Ya. E. Krasik, A. Dunaevsky, and J. Felsteiner, Phys. Plasmas 8, 2466 (2001), and references therein.

${ }^{4}$ I. Boscolo and S. Cialdi, J. Appl. Phys. 91, 6125 (2002).

${ }^{5}$ G. Rosenman, D. Shur, Ya. E. Krasik, and A. Dunaevsky, J. Appl. Phys. 88, 6109 (2000).

${ }^{6}$ K. Chirko, Ya. E. Krasik, A. Sayapin, J. Felsteiner, and V. Bernshtam, J. Appl. Phys. 94, 1420 (2003).

${ }^{7}$ A. Dunaevsky, K. Chirko, Ya. E. Krasik, J. Felsteiner, and V. Bernshtam, J. Appl. Phys. 90, 4108 (2001).

${ }^{8}$ A. Dunaevsky and N. J. Fisch, J. Appl. Phys. (submitted).

${ }^{9}$ K. Chirko, Ya. E. Krasik, J. Felsteiner, and A. Sternlieb, J. Appl. Phys. 92, 5691 (2002).

${ }^{10}$ K. Teranishi, S. Suzuki, and H. Itoh, Jpn. J. Appl. Phys., Part 1 40, 5766 (2001).

${ }^{11}$ H. Itoh, K. Teranishi, and S. Suzuki, IEEE Trans. Plasma Sci. 30, 124 (2002).

${ }^{12}$ M. Legentil, P. Postel, J. C. Thomas, and V. Puech, IEEE Trans. Plasma Sci. 23, 330 (1995).

${ }^{13}$ K. Bergmann, R. Lebert, J. Kiefer, and W. Neff, Appl. Phys. Lett. 71, 1936 (1997).

${ }^{14}$ G. D. Hobbs and J. A. Wesson, Plasma Phys. 9, 85 (1967).

${ }^{15}$ H. C. Miller, IEEE Trans. Electr. Insul. 24, 765 (1989).

${ }^{16}$ A. S. Pillai and R. Hackam, J. Appl. Phys. 53, 2983 (1982).

${ }^{17}$ R. A. Anderson and J. P. Brainard, J. Appl. Phys. 51, 1414 (1980).

${ }^{18}$ A. Dunaevsky, Y. Raitses, and N. J. Fisch, Phys. Plasmas 10, 2574 (2003).

${ }^{19}$ K. U. Riemann and L. Tsendin, J. Appl. Phys. 90, 5487 (2001).

${ }^{20}$ Yu. P. Raizer, Gas Discharge Physics (Springer-Verlag, Berlin, 1991), Chap. 6, p. 125.

${ }^{21}$ A. A. Avdienko and M. D. Malev, Vacuum 27, 643 (1977).

${ }^{22}$ S. P. Bugaev, A. M. Iskol'dskii, and G. A. Mesyats, Sov. Phys. Tech. Phys. 12, 1358 (1968).

${ }^{23}$ E. W. Gray, J. Appl. Phys. 58, 132 (1985).

${ }^{24}$ G. Masten, T. Muller, F. Hegeler, and H. Krompholz, IEEE Trans. Plasma Sci. 22, 1034 (1994). 


\section{External Distribution}

Plasma Research Laboratory, Australian National University, Australia

Professor I.R. Jones, Flinders University, Australia

Professor João Canalle, Instituto de Fisica DEQ/IF - UERJ, Brazil

Mr. Gerson O. Ludwig, Instituto Nacional de Pesquisas, Brazil

Dr. P.H. Sakanaka, Instituto Fisica, Brazil

The Librarian, Culham Laboratory, England

Mrs. S.A. Hutchinson, JET Library, England

Professor M.N. Bussac, Ecole Polytechnique, France

Librarian, Max-Planck-Institut für Plasmaphysik, Germany

Jolan Moldvai, Reports Library, Hungarian Academy of Sciences, Central Research Institute for Physics, Hungary

Dr. P. Kaw, Institute for Plasma Research, India

Ms. P.J. Pathak, Librarian, Institute for Plasma Research, India

Ms. Clelia De Palo, Associazione EURATOM-ENEA, Italy

Dr. G. Grosso, Instituto di Fisica del Plasma, Italy

Librarian, Naka Fusion Research Establishment, JAERI, Japan

Library, Laboratory for Complex Energy Processes, Institute for Advanced Study, Kyoto University, Japan

Research Information Center, National Institute for Fusion Science, Japan

Dr. O. Mitarai, Kyushu Tokai University, Japan

Dr. Jiangang Li, Institute of Plasma Physics, Chinese Academy of Sciences, People's Republic of China

Professor Yuping Huo, School of Physical Science and Technology, People's Republic of China

Library, Academia Sinica, Institute of Plasma Physics, People's Republic of China

Librarian, Institute of Physics, Chinese Academy of Sciences, People's Republic of China

Dr. S. Mirnov, TRINITI, Troitsk, Russian Federation, Russia

Dr. V.S. Strelkov, Kurchatov Institute, Russian Federation, Russia

Professor Peter Lukac, Katedra Fyziky Plazmy MFF UK, Mlynska dolina F-2, Komenskeho Univerzita, SK-842 15 Bratislava, Slovakia

Dr. G.S. Lee, Korea Basic Science Institute, South Korea

Institute for Plasma Research, University of Maryland, USA

Librarian, Fusion Energy Division, Oak Ridge National Laboratory, USA

Librarian, Institute of Fusion Studies, University of Texas, USA

Librarian, Magnetic Fusion Program, Lawrence Livermore National Laboratory, USA

Library, General Atomics, USA

Plasma Physics Group, Fusion Energy Research Program, University of California at San Diego, USA

Plasma Physics Library, Columbia University, USA

Alkesh Punjabi, Center for Fusion Research and Training, Hampton University, USA

Dr. W.M. Stacey, Fusion Research Center, Georgia Institute of Technology, USA

Dr. John Willis, U.S. Department of Energy, Office of Fusion Energy Sciences, USA

Mr. Paul H. Wright, Indianapolis, Indiana, USA 
The Princeton Plasma Physics Laboratory is operated by Princeton University under contract with the U.S. Department of Energy.

\author{
Information Services \\ Princeton Plasma Physics Laboratory \\ P.O. Box 451 \\ Princeton, NJ 08543
}

Phone: 609-243-2750

Fax: 609-243-2751

e-mail: pppl_info@pppl.gov

Internet Address: http://www.pppl.gov 